\title{
Análisis coste-efectividad del tratamiento con Ramipril de pacientes con alto riesgo de padecer eventos cardiovasculares en España
}

\author{
W. M. HART, C. RUBIO-TERRÉS*, I. MARGALET FERNÁNDEZ*, J. R. GONZÁLEZ \\ JUANATEY** \\ EcoStat Consulting Group, San Sebastián. *Departamento Científico. Aventis Pharma, \\ S.A. Madrid. **Servicio de Cardiología. Complejo Hospitalario de Santiago. Santiago de \\ Compostela.
}

\begin{abstract}
COST-EFFECTIVENESS ANALYSIS OF RAMIPRIL TREATMENT OF PATIENTS AT HIGH-RISK OF CARDIOVASCULAR EVENTS IN SPAIN
\end{abstract}

\section{RESUMEN}

Introducción y objetivos: Se calculó el coste-efectividad de añadir Ramipril al tratamiento habitual en pacientes con alto riesgo de padecer eventos cardiovasculares.

Métodos: Se hizo un modelo farmacoeconómico retrospectivo. Los datos clínicos se obtuvieron de un ensayo clínico aleatorizado en 9.297 pacientes (estudio HOPE). La información sobre la utilización de recursos y sus costes asociados se obtuvo de fuentes españolas. La perspectiva del análisis fue la del Sistema Nacional de Salud y se concentró en los costes de los fármacos y las complicaciones principales y secundarias de los eventos cardiovasculares.

Resultados: El coste incremental por año de vida ganado en el caso básico fue de 10.329 euros (tasa de descuento del 6\%). Un análisis de sensibilidad mostró que los resultados fueron más sensibles a los cambios en la estimación de la esperanza de vida después de la terminación del ensayo que a cualquiera otra variable. El número de casos de infarto, ictus o muerte cardiovascular que se podría evitar en cinco años oscila entre 19.000 y 39.000 .

Conclusiones: El uso de Ramipril en adición al tratamiento convencional en pacientes con alto riesgo cardiovascular es coste-efectivo y representaría menos gasto por año de vida ganado que varios tratamientos actualmente financiados por el Sistema Nacional de Salud.

PALABRAS CLAVE: Análisis coste-beneficio. Enfermedades cardiovasculares. Inhibidores enzima conversiva. Ramipril.

\section{ABSTRACT}

Introduction and objectives: The cost-effectiveness of adding rami pril to the regular treatment of patients at high risk of suffering cardio vascular events was calculated.

Methods: A retrospective pharmacoeconomic model was used. The clinical data were obtained from a randomized clinical trial of 9,297 patients (the HOPE study). Information about resource use and the associated costs was obtained from Spanish sources. The analysis was under taken from the perspective of the National Health System, focusing on drug costs and the primary and secondary complications of cardiovascu lar events.

Results: The incremental cost per life-year gained in the base case was 10,329 euros (6\% discount rate). A sensitivity analysis showed that the results were more sensitive to changes in estimated post-trial life expectancy than to any other variable. The estimated number of cases of myocardial infarction, stroke, or cardiovascular death prevented in five years ranged from 19,000 to 39,000 .

Conclusions: The addition of Ramipril to the conventional treatment of patients at high cardiovascular risk is cost-effective and less expensive per life-year gained than several treatments currently financed by the Spanish National Health System.

KEY WORDS: Cost-benefit analysis. Cardiovascular diseases. Angio tensin-converting-enzyme inhibitor. Ramipril.

Hart WM, Rubio-Terrés C, Margalet Fernández I, González Juanatey JR. Análisis coste-efectividad del tratamiento con Ramipril de pacientes con alto riesgo de padecer eventos cardiovasculares en España. An Med Interna (Madrid) 2002; 19: 515-520.

\section{INTRODUCCIÓN}

Durante los últimos años, varios estudios han demostrado que la utilización de los inhibidores de la enzima conversiva de la angiotensina (IECAs) es coste-efectiva (1-8). Sin embargo, existe cierta controversia acerca de los resultados de un meta-análisis de tres estudios (9-11), según el cual el tratamiento con IECAs redujo el riesgo de infarto de miocardio un $23 \%$ (9-11). Por este motivo, se decidió evaluar en el estudio HOPE (12) (un ensayo clínico en el que se aleatorizaron 9.297 pacientes con alto riesgo de sufrir eventos cardiovasculares) los efectos del tratamiento durante cinco años con un IECA como Ramipril. Según sus resultados, la asociación del tratamiento con Ramipril al tratamiento habitual dio lugar, en comparación con el tratamiento convencional solo, a reducciones significativas del riesgo de padecer complicaciones cardiovasculares. Se redujo un $26 \%$ el riesgo relativo de mortalidad cardiovascular $(\mathrm{P}<0,001)$, un $20 \%$ el riesgo de infarto de miocardio $(\mathrm{P}<0,001)$, un $32 \%$ el riesgo de ictus cerebral $(\mathrm{P}<0,001)$ y un $16 \%$ el riesgo de muerte por cualquier causa

Trabajo aceptado: 6 de junio de 2002

Correspondencia: Carlos Rubio Terrés. Aventis Pharma S.A. C/ Martínez Villergas, 52, 28027 Madrid. e-mail: carlos.rubio@aventis.com. 
$(\mathrm{P}=0,005)$. Así mismo, se redujeron significativamente los riesgos de revascularización, parada cardíaca, insuficiencia cardíaca y de complicaciones relacionadas con la diabetes (12). Estos beneficios del tratamiento con Ramipril, así como el coste del propio tratamiento, conllevan una repercusión económica. En consecuencia, parece lógico extender el análisis del estudio HOPE incorporando las dimensiones económicas que corresponderían a la práctica clínica en España. El objetivo del presente estudio fue averiguar el impacto de las reducciones en la morbilidad y la mortalidad debidas al tratamiento con Ramipril y comparar, desde un punto de vista económico, esta intervención con las actualmente financiadas por el Sistema Nacional de Salud (SNS).

Se dispone de algunos estudios preliminares realizados en otros países con un planteamiento similar al nuestro. En un análisis canadiense (13) se concluyó que el tratamiento con Ramipril sería dominante (más efectivo y menos costoso que la opción de no tratar), mientras que en un estudio realizado en el Reino Unido (14) se dedujo que la utilización de Ramipril en combinación con el tratamiento convencional daría lugar a un mayor gasto pero dentro de los límites de lo que se considera una buena utilización de recursos limitados. Estas diferencias pueden ser explicadas por diferencias en la metodología utilizada y en los costes relativos de los dos sistemas sanitarios. Por lo tanto, se decidió realizar un estudio específico en España para identificar las implicaciones económicas en nuestro ámbito.

\section{MATERIAL Y MÉTODOS}

\section{MODELO FARMACOECONÓMICO}

Se ha desarrollado un modelo para probar la hipótesis de que el uso adicional de Ramipril no es solo ventajoso clínicamente, sino que también representa una opción coste-efectiva en la prevención de eventos cardiovasculares en los pacientes de alto riesgo.

Se ha supuesto una duración de tratamiento de cinco años (conforme a la duración del ensayo clínico HOPE) y una supervivencia adicional de 4,3 años basada en los últimos datos de la esperanza de vida de la población española (15) teniendo en cuenta la edad media de los pacientes del estudio HOPE (66 \pm 7 años) y su distribución según el sexo (mujer: varón; 26,7:73,3). Esta variable, la esperanza de vida, ha sido objeto de un análisis de sensibilidad dada la naturaleza incierta de su valor.

Se ha realizado un análisis coste-efectividad incremental desde la perspectiva del SNS y, por tanto, no se han incorporado costes indirectos (laborales) que, de cualquier modo, serían mínimos dada la edad media de la población al inicio del ensayo clínico.

El modelo farmacoeconómico se basó en el análisis secundario de los datos existentes. Los parámetros de costes considerados fueron los siguientes: a) la terapia con Ramipril, teniendo en cuenta la reducción del cumplimiento del tratamiento durante cada uno de los cinco años; b) los costes de los eventos cardiovasculares principales: infarto de miocardio e ictus cerebral; c) los costes de las consecuencias secundarias (revascularización, hospitalización por angina, complicaciones relacionadas con la diabetes y la hospitalización debida a la insuficiencia cardiaca).
La medida de la efectividad se expresó como años de vida ganados (AVGs), estimados a partir de los datos publicados del estudio HOPE (considerando el área entre las curvas de supervivencia). Se estimó el coste-efectividad incremental debido a la adición de Ramipril al tratamiento convencional según la siguiente formula:

$$
\mathrm{ACEI}=(\Delta \mathrm{CR}+\Delta \mathrm{CIM}+\Delta \mathrm{CEC}+\Delta \mathrm{CCS}) / \Delta \mathrm{AVG}
$$

Donde ACEI es el análisis coste-efectividad incremental, comparando la adición de Ramipril a la terapia habitual con la terapia habitual solamente; $\Delta \mathrm{CR}$ es el coste adicional de Ramipril, teniendo en cuenta las tasas anuales de cumplimiento terapéutico; $\triangle \mathrm{CIM}$ es el coste adicional del infarto de miocardio (una reducción sería negativa); $\Delta$ CECes el coste adicional del ictus cerebral (una reducción sería negativa); $\Delta \mathrm{CCS}$ son los costes adicionales debidos a las consecuencias secundarias del uso de Ramipril (una reducción sería negativa); finalmente, $\triangle \mathrm{AVG}$ son los años de vida ganados como consecuencia de la intervención con Ramipril.

\section{ESTIMACIÓN DE LA EFICACIA}

Se han utilizado los datos de mortalidad (por todas las causas) del estudio HOPE (12). La información disponible a partir de la publicación es limitada. Por este motivo, fue necesario hacer una aproximación a la distribución de la supervivencia para poder estimar diferencias anuales en la supervivencia y poder hacer proyecciones más allá de la duración del ensayo clínico. Dicha aproximación se hizo considerando una relación exponencial entre la mortalidad y el tiempo, mediante la adopción del método "Declining Exponential Approximation of Life Expectancy" (DEALE) (16). El método "DEALE" se considera apropiado para este tipo de análisis, suponiendo una tasa de mortalidad constante que puede ser una buena aproximación cuando el periodo considerado no es demasiado largo.

\section{RECURSOS Y COSTES}

Todos los costes fueron actualizados al año 2.000 utilizando el índice de precios al consumo (IPC) del Instituto Nacional de Estadística (www.ine.es). En el caso del año 2.000 se utilizó la tasa interanual hasta el mes de octubre (4\%).

\section{RAMIPRIL}

Se ha supuesto un dosis diaria de $10 \mathrm{mg}$ durante todo el periodo del estudio. En el año 2.000 un envase de Ramipril con 28 comprimidos de $5 \mathrm{mg}$ cuesta 17,34 euros (17) (incluyendo el IVA del 4\%). Esto representa un coste anual de 452 euros.

\section{Cumplimiento terapéutico con Ramipril}

En el estudio HOPE se registró el cumplimiento terapéutico con Ramipril al final de cada año. El porcentaje de pacientes que recibió $10 \mathrm{mg}$ de Ramipril por día fue del $82,9 \%$ al final del primer año, 74,6\% al final del segundo año, 70,9\% después de tres años, 62,4\% después de cuatro años y 65,0\% 
al final del estudio. Se ha supuesto un abandono lineal del tratamiento en cada periodo de un año, y se ha utilizado el promedio aritmético de la utilización al principio y al final de cada periodo para estimar la utilización de Ramipril durante ese periodo. Estos datos están resumidos en la tabla I, junto con los costes totales por paciente en cada año.

Se ha supuesto que no hay otros costes adicionales (visitas, pruebas, etc.) asociados con la administración de Ramipril, dado que se está considerando un tratamiento adicional y que no se está comparando con una opción sin tratamiento.

\section{Costes de las complicaciones (Tabla II)}

1. Infarto de miocardio: los costes de un infarto de miocardio están basados en un promedio de los valores de los Grupos Relacionados por el Diagnóstico (GRDs) 121 y 122 de tres fuentes distintas (18-20). El valor promedio del coste actualizado al año 2000 es de 5.191 euros.

2. Ictus cerebral: en un estudio de Carod-Artel y cols. (21) se analizaron los costes directos del ictus cerebral durante el primer año. El coste actualizado al año 2000 fue de 4.821 euros.

3. Revascularización: se consultó con un cardiólogo del Hospital San Pablo de Barcelona, quien describió el proceso y los recursos utilizados para la realización de este tipo de intervención desde el ingreso hasta el alta: la estancia hospitalaria, la estancia en la UCI, los materiales sanitarios, etc, a los cuales se asignaron los correspondientes costes unitarios (22), obtenidos de una base de datos de costes sanitarios españoles.

4. Angina inestable: el coste de la angina (ICD 413.X y 411.1) se estimó considerando un promedio de 9,55 días de estancia (23), con un coste total de 3.351 euros.

5. Diabetes: en un estudio de Monereo y cols. (24) se utilizaron los datos económicos aportados por el Departamento de Gestión del Hospital Universitario de Getafe (Madrid). Para el GRD 294 (diabéticos mayores de 35 años) se contabilizaron 94 estancias y una estancia media de 7,2 días. El gasto por proceso (desde el ingreso al alta) fue de 2.782 euros en 2000.

6. Insuficiencia cardiaca: en el caso de la insuficiencia cardiaca (ICD 428.X) se estimó un promedio de 11,6 días de estancia (23) con un coste total de 4.070 euros en 2000.

Dado que no se dispone de datos acerca de la distribución temporal de las complicaciones, se ha supuesto que su distribución esté ponderada al número de supervivientes en cada periodo anual. Por lo tanto, con este criterio se observa un número descendiente de complicaciones por año a causa de la reducción en el número total de pacientes al final de cada año.
TABLA II

\section{CO STES DE LAS CO M PLICACIO NES PRINCIPALES Y} SECUNDARIAS

\begin{tabular}{lcc}
\hline Complicación & $\begin{array}{c}\text { Coste (euros; actualizado } \\
\text { al año 2000) }\end{array}$ & Fuente \\
\hline Infarto de miocardio & 5.191 & $18-20$ \\
Ictus cerebral & 4.821 & 21 \\
Revascularización & 11.101 & 22 \\
Angina & 3.351 & 23 \\
Diabetes & 2.782 & 24 \\
Insuficiencia cardiaca & 4.070 & 23 \\
\hline
\end{tabular}

\section{TASA DE DESCUENTO}

En el caso básico se utilizó una tasa de descuento de $6 \%$ para los costes y los efectos de las intervenciones. Esta tasa es la propuesta para las evaluaciones económicas en España (25).

\section{ANÁLISIS DE SENSIBILIDAD}

Como es habitual en los análisis farmacoeconómicos, se han variado los valores claves del análisis para determinar su influencia sobre los resultados finales. En concreto se modificaron dos variables: el número de años de supervivencia después de finalizar el ensayo clínico HOPE y el coste de las complicaciones de los eventos cardiovasculares.

\section{ANÁLISIS DE NNTS}

Aparte del análisis económico en términos de coste por Años de Vida Ganados (AVGs) se ha intentado establecer el número de eventos que se podrían evitar por utilizar Ramipril. El punto de partida para este cálculo es el número de pacientes que se tiene que tratar (en inglés "Number Needed to Treat" (NNT)) para evitar un evento. Se ha estimado que la población con una edad mayor de 54 años es de 10.625.014 en el año 2001 (26) y que la proporción de esta población con alto riesgo de sufrir un evento cardiovascular en el caso básico es del 10\% (27-38). Esta última estimación se basa en una revisión de estudios españoles de referencia en los que se consideraron los mismos factores de riesgo de los pacientes del estudio HOPE.

\section{TABLA I}

UTILIZACIÓ N Y COSTE ANUAL DE RAM IPRIL (10 M G/DÍA) PO R PACIENTE (EUROS)

\begin{tabular}{lccc}
\hline Periodo & Utilización anual (\%) & $\begin{array}{c}\text { Coste anual } \\
\text { (sin descontar) }\end{array}$ & $\begin{array}{c}\text { Coste anual } \\
\text { (tasa de descuento de 6\%) }\end{array}$ \\
\hline Durante el primer año & 91,5 & 414 & 414 \\
Durante el segundo año & 78,8 & 356 & 336 \\
Durante el tercer año & 72,8 & 329 & 293 \\
Durante el cuarto año & 66,7 & 301 & 253 \\
Durante el quinto año & 63,7 & 288 & 228 \\
Coste total (5 años) & & 1.688 & 1.524 \\
\hline
\end{tabular}


En primer lugar, la prevalencia global de angina en España según el estudio PANES es del 7,5\% (27). Esta prevalencia es congruente con la observada en estudios realizados en países vecinos (28-30). En el mencionado estudio, que no incluyó a mayores de 74 años, la prevalencia aumentó con la edad $(8,0$ y 8,8\% en varones y mujeres de 65-74 años respectivamente).

En segundo lugar, la prevalencia de hipertensión arterial (HTA) oscila entre el 20 y el 50\% según la población considerada y la definición utilizada de HTA (31-33). En el mismo estudio PANES se observó una prevalencia de HTA autodeclarada del $31,1 \%$ que puede representar una infravaloración de la prevalencia real dado que solamente algo más de la mitad de los hipertensos conocen su enfermedad (33).

Por último, se han revisado los datos de la prevalencia de la diabetes mellitus (DM). En términos generales, los estudios publicados indican una prevalencia global de la DM conocida de un $2,8-3,9 \%$ de la que aproximadamente el $10 \%$ corresponde a DM tipo I (34). Esta cifra se eleva con la edad, llegando al $10-18 \%$ en los mayores de 65 años (35-37). Trasladando estos porcentajes a números de pacientes, supone una cifra total de 1-1,5 millones de diabéticos (38) de los cuales la mayoría es de una edad superior a los 55 años. Por lo tanto, parece justificable estimar que el tratamiento con Ramipril estaría indicado en un $10 \%$ de la población mayor de 54 años. Se reconoce la naturaleza aproximada de estos cálculos que, sin embargo, añaden datos importantes al micro-análisis del coste por AVG, extendiéndolo a un escenario más amplio. En el análisis de sensibilidad se ha considerado un intervalo entre el 5 y el $15 \%$.

\section{RESULTADOS}

En las tablas III y IV se exponen los resultados del análisis del caso básico y las consecuencias del análisis de sensibilidad, respectivamente. El coste incremental por año de vida ganado oscila aproximadamente entre 8.300 y 10.300 euros según la tasa de descuento ( 0 y $6 \%$ respectivamente). Los años de vida ganados con la opción de Ramipril serían 0,14 ( $\sin$ descontar), disminuyendo a 0,10 como consecuencia del proceso de descontar los beneficios. Los costes (durante un periodo de cinco años) son aproximadamente un $30 \%$ mayores en el caso de añadir Ramipril al tratamiento habitual.
La tabla IV nos muestra la sensibilidad de los resultados al modificar el supuesto de supervivencia de los pacientes que llegan al final del ensayo (el 89,6\% en el caso de Ramipril y el $87,8 \%$ en el caso del tratamiento convencional). Al extender la esperanza de vida de 5,33 a 8 años se obtiene un coste incremental por año de vida ganado de unos 8.300 euros, mientras que la reducción de esta variable a dos años aumenta el coste incremental por año de vida ganado a 16.000 euros. Por el contrario, se observa que los cambios en los costes de las complicaciones $( \pm 25 \%)$ tienen poco impacto en los resultados.

En la tabla V, se presentan los NNTs durante un periodo de 5 años de los eventos principales del análisis. Se observa que hay mucha variación, oscilando entre 27 pacientes tratados para evitar un infarto, ictus o muerte cardiovascular hasta los casi 460 pacientes que deberían ser tratados para evitar un caso de hospitalización por angina inestable.

Sin embargo, el significado de estos datos reside en el tamaño de la población que potencialmente puede beneficiarse de la intervención con Ramipril. En la tabla VI se resumen algunas estimaciones suponiendo, de manera conservadora, que el $10 \%$ de la población recibiese el tratamiento.

\section{DISCUSIÓN}

Los resultados del estudio HOPE indican que la utilización de Ramipril en pacientes con alto riesgo de padecer eventos cardiovasculares está asociada con un aumento en la supervivencia y una reducción en el número de complicaciones, siendo numerosos los pacientes que podrían beneficiarse de tales efectos. Los resultados de este análisis demuestran que, además, Ramipril es coste-efectivo utilizando criterios internacionales para la definición de una intervención costeefectiva.

Esta no es la primera evaluación económica de Ramipril, pero sí una de las primeras en utilizar los datos de eficacia del estudio HOPE. Los resultados obtenidos son inferiores (más coste-efectivos) a los descritos para algunos tratamientos actualmente financiados por el SNS. Se ha sugerido que un tratamiento con un coste por año de vida ganado de menos de 50.000 dólares (unos 43.000 euros) representa una buena utilización de recursos limitados. De hecho, se están utilizando fármacos hipolipemiantes financiados por el SNS con un cos-

TABLA III

RESULTADOS DEL CASO BÁSICO (ESPERANZA DE VIDA 5,3 AÑOS DESPUÉS DEL ENSAYO CLÍNICO)

\begin{tabular}{ccccc}
\hline & Sin Descontar & \multicolumn{2}{c}{ Descontado (6\%) } \\
\hline $\begin{array}{c}\text { Ramipril }+ \\
\text { tratamiento } \\
\text { habitual }\end{array}$ & $\begin{array}{c}\text { Tratamiento } \\
\text { habitual }\end{array}$ & Diferencia & $\begin{array}{c}\text { Ramipril }+ \\
\text { tratamiento } \\
\text { habitual }\end{array}$ & $\begin{array}{c}\text { Tratamiento } \\
\text { habitual }\end{array}$ \\
\end{tabular}

\begin{tabular}{|c|c|c|c|c|c|c|}
\hline $\begin{array}{l}\text { Coste } \\
\text { estimado por } \\
\text { paciente (euros) }\end{array}$ & 4.841 & 3.661 & 1.180 & 4.347 & 3.280 & 1.067 \\
\hline $\begin{array}{l}\text { Años de vida } \\
\text { ganados }\end{array}$ & 9,51 & 9,37 & 0,14 & 7,39 & 7,29 & 0,10 \\
\hline $\begin{array}{l}\text { Análisis coste- } \\
\text { efectividad } \\
\text { incremental }\end{array}$ & & & 8.266 & & & 10.329 \\
\hline
\end{tabular}


TABLA IV

ANÁLISIS DE SENSIBILIDAD DEL ANÁLISIS COSTE-EFECTIVIDAD INCREM ENTAL

\begin{tabular}{|c|c|c|c|c|c|}
\hline & & \multicolumn{4}{|c|}{ Años de supervivencia después del final del ensayo } \\
\hline Sin otros cambios & & $\begin{array}{c}2 \\
16.152\end{array}$ & $\begin{array}{c}4 \\
11.945\end{array}$ & $\begin{array}{c}6 \\
9.697\end{array}$ & $\begin{array}{c}8 \\
8.306\end{array}$ \\
\hline Coste de las complicaciones & $\begin{array}{l}+25 \% \\
-25 \%\end{array}$ & $\begin{array}{l}14.423 \\
17.881\end{array}$ & $\begin{array}{l}10.666 \\
13.223\end{array}$ & $\begin{array}{r}8.659 \\
10.735\end{array}$ & $\begin{array}{l}7.417 \\
9.195\end{array}$ \\
\hline
\end{tabular}

TABLA $V$

NNTS SEGÚN EL ESTUDIO HOPE

\begin{tabular}{lc}
\hline Evento & $\begin{array}{c}\text { NNTs con } \\
\text { Ramipril para } \\
\text { evitar un caso }\end{array}$ \\
\hline Infarto, ictus, muerte CV. & 27 \\
Muerte CV & 50 \\
Infarto & 43 \\
Ictus cerebral & 67 \\
M uerte por cualquier causa & 54 \\
Revascularización & 43 \\
Hospitalización por angina inestable & 458 \\
Complicación relacionada con diabetes & 86 \\
Hospitalización debida a insuficiencia cardíaca & 248 \\
\hline
\end{tabular}

te por AVG de hasta 69.700 euros (39). En otro estudio español en el que se hizo una evaluación económica de las intervenciones utilizadas para la prevención de las enfermedades coronarias (40) se obtuvieron ratios de coste-efectividad que oscilaron entre 3.000 euros y 240.000 euros. En consecuencia, los resultados de nuestro estudio indican que la combina- cion de Ramipril es coste-efectiva en nuestro entorno, considerando tanto el consenso internacional como la comparación con otros tratamientos financiados en España.

La utilización de los IECAs se ha ido ampliando a lo largo de los últimos años a nuevas indicaciones como consecuencia de los resultados de los ensayos clínicos llevados a cabo (41). El ratio de coste-efectividad obtenido, así como las repercusiones sanitarias estimadas, debe invitar a una reflexión seria sobre la conveniencia de emplear Ramipril en los pacientes con alto riesgo de padecer eventos cardiovasculares.

El presente estudio tiene varias limitaciones que deben ser tenidas en cuenta. En primer lugar, es un modelo basado en los resultados de un ensayo clínico doble ciego y aleatorizado, no en un ensayo clínico pragmático, por lo que sus resultados no pueden considerarse directamente extrapolables a la práctica clínica. Por otra parte, los datos disponibles del estudio HOPE son muy resumidos y, por lo tanto, ha sido necesario hacer estimaciones acerca de la distribución de las curvas de supervivencia y la distribución de los eventos adversos durante el periodo del ensayo. También se han hecho estimaciones sobre la esperanza de vida después de la terminación del mismo. No obstante, si no se tuviera en cuenta este tiempo adicional como consecuencia de la utilización de Ramipril existiría la posibilidad de una subestimación importante de los beneficios del IECA. La pregunta que el lector podría hacerse es la siguiente: ¿es razonable esperar que los pacientes que llegan al final del ensayo, con una edad promedio de 71 años, podrían vivir durante cinco años más?. Esta duración es inferior a la

TABLA VI

IM PLICACIO NES CLÍNICAS DE LOS NNTS DURANTE CINCO AÑOS SEGÚN EL ESTUDIO HO PE

Evento Casos evitados

\begin{tabular}{lccc}
\hline & $\begin{array}{c}\text { Mínimo } \\
(531.251)^{*}\end{array}$ & $\begin{array}{c}\text { Caso básico } \\
(1.062 .501)^{*}\end{array}$ & $\begin{array}{c}\text { M áximo } \\
(1.593 .752)^{*}\end{array}$ \\
\hline Infarto, ictus, muerte CV. & 19.676 & 39.352 & 59.028 \\
M uerte CV & 10.652 & 21.250 & 31.875 \\
Infarto & 12.355 & 24.710 & 37.065 \\
Ictus cerebral & 7.929 & 15.858 & 23.787 \\
M uerte por cualquier causa & 9.838 & 19.676 & 29.514 \\
Revascularización & 12.355 & 24.710 & 37.065 \\
Hospitalización por angor inestable & 1.160 & 2.320 & 3.480 \\
Complicación relacionada con diabetes & 6.177 & 12.354 & 18.531 \\
Hospitalización debida a insuficiencia cardíaca & 2.142 & 4.248 & 6.372 \\
\hline
\end{tabular}

* Pacientes potenciales. 
considerada en un estudio similar realizado en el Reino Unido (14), en el que se estimó que la esperanza de vida después del ensayo sería de 5,89 años. Por lo tanto, nuestra estimación (basada en la esperanza de vida de los españoles, que es mayor que la de los británicos) parece conservadora.

En el caso básico del estudio, la utilización adicional de Ramipril implicaría un gasto adicional de unos 10.000 euros por año de vida ganado y representaría una opción coste-efectiva según los consensos internacionales y considerando que Ramipril es más coste-efectivo que otras intervenciones actualmente financiadas por el SNS. Ramipril es clínicamente beneficioso y económicamente coste-efectivo en los pacientes con alto riesgo de padecer eventos cardiovasculares, siendo considerable el impacto sanitario de una potencial intervención preventiva.

\section{Bibliografía}

1. Le Pen $\mathrm{C}$, et al. Cost-effectiveness analysis of captopril treatment after myocardial infarction. Arch Mal Coeur Vaiss 1994; 87: 775-81.

2. Tsevet $\mathbf{J}$, et al. Cost-effectiveness of captopril therapy after myocardial infarction. J Am Coll Cardiol 1995; 26: 914-9.

3. Michel BC, et al. Economic aspects of treatment with captopril for patients with asymptomatic left ventricular dysfunction in the Netherlands. Eur Heart J 1996; 17: 731-40.

4. Hummel S, et al. An economic analysis of the survival and ventricular enlargement (SAVE) study. PharmacoEconomics 1997; 12: 182-92.

5. Le Pen C, et al. A cost-effectiveness análisis of tradolapril in postinfarction patients with left ventricular dysfunction. PharmacoEconomics 1998; 14: 49-58.

6. Szucs TD et al. Cost-effectiveness of captopril after myocardial infarction. Med Klinic 1996; 91: 112-8

7. Andresson F, et al. ACE inhibitors and heart failure: the consequences of undersubscribing. PharmacoEconomics 1999; 15 (6): 535-50.

8. McMurray JJV, et al. Cost-effectiveness of different ACE inhibitor treatment scenarios post-myocardial infarction. Eur Heart J 1997; 18: 1411-5.

9. Loon EM, et al. Emerging role of ACE inhibitors in cardiac and vascular protection. Circulation 1994; 90: 2056-69.

10. Yusuf S et al. Effect of enalapril on myocardial infarction and unstable angina in patients with low ejection fractions. Lancet 1992; 340: 1173-8.

11. Pfeffer MA, et al. Effect of captopril on mortality and morbidity in patients with left ventricular dysfunction after myocardial infarction: results of the Survival and Ventricular Enlargement trial. N Engl J Med 1992; 327: 669-77.

12. The Heart Outcomes Prevention Evaluation Study Investigators. Effects of an angiotensin-converting-enzyme inhibitor, Ramipril, on cardiovascular events in high-risk patients. N Engl J Med 2000; 342: 145-53.

13. Lamy A, Yusuf S, Pogue J, Gafni A. Ramipril for high-risk patients: the economic implications of the HOPE study. Canadian Cardiovascular Society, poster, October 2000.

14. Bachhouse M, Richter A, Gaffney L. Economic evaluation of Ramipril in the treatment of patients at high risk for cardiovascular events. Value in Health 2000; 3 (5): 311.

15. Instituto Nacional de Estadística, Base de datos TEMPUS. Madrid 2000.

16. Beck RJ, Kassirer JP, Pauker SG . A convenient approximation of life expectancy (the DEALE). Am J Med 1982; 73: 883-98.

17. Vademécum Internacional 2000. MediMedia Medicom S.A. Madrid 2000.

18. Hospital Arquitecto Marcide-Novoa Santos. Memoria Anual 1993.

19. Casas M. Los grupos relacionados con el diagnóstico. Ed Masson, Barcelona 1991.

20. Jiménez J. La determinación de los costes por proceso. Validación de los pesos asociados a los GRD mediante la aplicación de los costes reales obtenidos del Sistema de Información Económica (SIE). XV Jornadas de Economía de la Salud. Gaceta Sanitaria 1995; 48 (9).

21. Carod-Artel FJ, et al. Direct costs of cerebrovascular disease during the first-year of follow-up. Rev Neuro 1999; 8: 1123-30.

22. Hart $\mathrm{W}$ et al. The cost of coronary bypass grafting and PTCA in Spain. Soikos, Barcelona 1994.

23. CMBD. Mortalidad Hospitalaria en Catalunya. Barcelona 1992.
24. Monereo S, Pavón I, Vega B, Elviro R. Diabetes mellitus: aproximación al coste por proceso aplicado al paciente diabético ingresado. Endocrinología 1997; 44 (9): 319-25.

25. Propuesta de estimación de algunos aspectos metodológicos de los análisis coste-efectividad y coste-utilidad en la evaluación de tecnologías y programas sanitarios. Badia X, Rovira J. Evaluación económica de medicamentos. 1994 Luzán 5, S.A. de Ediciones.

26. Base de datos TEMPUS. Instituto Nacional de Estadística, Madrid 2001.

27. Cosín J, Asín E, Marrugat J, Elosua R, Arós F, De los Reyes M, et al, for the PANES Study group. Prevalence of angina pectoris in Spain. Eur J Epidemiology 1999; 15: 323-30

28. LaCroix AZ, Guraalnik JM, Curb DJ, Wallace RB, Ostfield AM, Hennekens $\mathrm{CH}$. Chest pain and coronary heart disease mortality among older men and women in three communities. Circulation 1990; 81: 43746.

29. Kuller L, Borhani N, Furberg C, Gardin J, Manolio T, O’Leary D et al. Prevalence of subclinical atherosclerosis and cardiovascular disease and association with risk factors in the Cardiovascular Health Study. Am J Epidemiol 1994; 139: 1164-79.

30. Krogh V, Trevisan M, Panico S, Farinaro E, Mancini M, Menotti A et al. Research Group ATS-RF2 of the Italian National Research Council. Prevalence and correlates of angina pectoris in the Italian nine communities study. Epidemiology 1991; 2: 26-32.

31. Masiá R, Pena A, Marrugat J, Sala J, Vila J, Pavesi M, et al, and the REGICOR Investigators. High prevalence of cardiovascular risk factors in Gerona, Spain, a province with low myocardial infarction incidence. J Epidemiol Común Health 1998; 52: 707-15.

32. Tresserras R, Pardell H. Prevalencia e importancia sanitaria de la hipertensión arterial. An Med Intern 1990;7:1-6.

33. Ministerio de Sanidad y Consumo y Sociedad-Liga Española para la lucha contra la hipertensión arterial. Control de la hipertensión arterial en España 1996. Madrid: Idepsa, 1996.

34. Goday A, Serrano Ríos M. Epidemiología de la diabetes mellitus en España. Revisión crítica y nuevas perspectivas. Med Clin (Barc) 1994; 102: 306-15.

35. Fernández Fernández MJ, Amo M, Jara A. La diabetes mellitus en la tercera edad. Prevalencia en una población de 576 individuos. Med Clin (Barc) 1987; 88: 490-3.

36. Bayo J, Sola C, Gracía F, Latorre PM, Vázquez JA. Prevalencia de la diabetes mellitus no insulinodependiente en Lejona (Vizcaya). Med Clin (Barc) 1993; 101: 609-12.

37. Franch J, Álvarez JC, Álvarez F, Diego F, Pablo M, Hernández R. El diagnóstico de la diabetes mellitus en los estudios epidemiológicos. Aten Primaria 1991; 8: 33-8.

38. Monereo S, Pavón I, Vega B, Elviro R. Diabetes mellitus: approximación al coste por proceso aplicado al paciente diabético ingresado. Endocrinología 1997; 44: 21-7

39. Plans Rubió P. Cost-effectiveness of pharmacologic treatments for the reduction of blood lipids. Med Clin (Barc) 1995; 105 (9): 327-33.

40. Plans-Rubió P. Cost-effectiveness of cardiovascular prevention programs in Spain. Int J Technol Assess Health Care 1998; 14 (2): 320-30.

41. Davie A. ACE Inhibitors after Myocardial Infarction: Clinical and Economic Considerations. PharmacoEconomics 2000; 17: 237-43. 